\title{
Leprosy research and elimination
}

\author{
S. K. NOORDEEN \\ Chennai, India
}

Summary The contribution of leprosy research to the progress being made toward elimination of leprosy has been critical. A major development in the promotion of leprosy research during the last 25 years has been the initiative taken by the WHO Special Programme for Research and Training in Tropical Diseases (TDR) through two of its scientific working groups, one on the immunology of leprosy (IMMLEP) and the other on the chemotherapy of leprosy (THELEP), which were set up in 1974 and 1976, respectively. IMMLEP and THELEP have greatly facilitated inputs from scientists not usually active in leprosy research. The coordinated efforts of IMMLEP and THELEP also facilitated goal-oriented research toward high-priority target areas such as an anti-leprosy vaccine and newer and better drug-combinations for the treatment of leprosy. Whereas the prospects for a leprosy vaccine appeared very promising in the early years, the first vaccine produced did not meet expectations for several reasons. Moreover, the possibility of using a vaccine in leprosy is not bright, because of both the technical problems as well as the reduced relevance of a vaccine at a time when leprosy is becoming less and less common. On the other hand, the modest expectations for newer and better drug combinations led to multi-drug therapy (MDT) for the control of leprosy. It is the introduction of MDT that is credited with the current global reduction of leprosy and the progress thus far made toward eliminating the disease as a public health problem. Nevertheless, many areas in leprosy, such as nerve damage, remain that require major research inputs in the future.

In recent years, tremendous progress has been made toward eliminating leprosy, progress that would not have been possible without the contribution of leprosy research. Until about 25 years ago, the global leprosy situation was rather dismal, and the situation of leprosy research was not very different. In those days, leprosy research tended to remain isolated, with very little input from exciting scientific developments in other areas. Also, leprosy research tended to be confined to clinical issues. Against this background, a major development took place in the mid-1970s, when the World Health Organization, together with the United Nations Development Program and the World Bank, set up the Special Programme for Research and Training in Tropical Diseases, known as TDR, with the aim of promoting goal-oriented, coordinated research in major tropical diseases including leprosy. The impetus to include leprosy came not only because leprosy was a major tropical disease affecting large populations, but also because of major developments in areas of basic research, particularly in immunology. The establishment of TDR followed closely on the discovery that the ninebanded armadillo was susceptible to infection by Mycobacterium leprae, and presented the possibility of producing large quantities of the organism for experimental work. The TDR programme set up scientific working groups in leprosy, one on the immunology of leprosy, known as IMMLEP, and the other on the chemotherapy of leprosy, known as THELEP. 
IMMLEP was particularly successful in projecting the possibility of conquering leprosy through the use of an anti-leprosy vaccine, the starting point for which were the large quantities of M. leprae it was able to obtain from armadillos maintained under its auspices. IMMLEP also looked into the possibility of developing diagnostic tests based on materials from M. leprae. In fact, IMMLEP's projection of leprosy as a problem with exciting possibilities for a solution led leprosy endemic countries to increase their attention to the control of leprosy. IMMLEP also made it possible for many scientists who were not involved in leprosy research to look upon leprosy as an interesting model, capable of providing solutions to many related problems. This influx of scientists from other scientific areas created a new situation, in which leprosy work was no longer isolated, and, as a result, it was able to benefit from scientific developments in other areas.

The THELEP scientific working group, which came into existence 2 years after IMMLEP, began with only modest expectations. However, THELEP was more related to present realities than was IMMLEP. Also, it was able to prepare a large number of scientists from developing, leprosy endemic countries to participate in leprosy research. This was greatly facilitated by the research-training component of the TDR programme.

In the course of time, it became apparent that conquering leprosy through the development of an anti-leprosy vaccine is a very long-term effort, with many obstacles in its path. Further, whereas IMMLEP developed many new diagnostic tools involving serology and skin tests, their usefulness in terms of specificity and sensitivity left much to be desired; these tools require further research. In the meantime, THELEP was able to identify many practical solutions with possibilities of immediate application to leprosy control. It was able to demonstrate in very dramatic fashion the importance of drug resistance of $M$. leprae to dapsone, then the most commonly used anti-leprosy drug, and the need to employ combinations of drugs for the treatment of leprosy. Thus came the concept of multidrug therapy (MDT) for successful treatment of the patients as individuals, and for control of leprosy in the community. Although the MDT regimens recommended by the WHO Study Group on Chemotherapy of Leprosy for Control Programmes in 1981 were based on a combination of drugs not yet widely tested, the basis for the recommendation came from extensive analysis and discussions within THELEP. Today, in retrospect, we recognize the recommendations of the 1981 WHO Study Group as a historic landmark that enabled the tremendous progress towards leprosy elimination that has been realized in recent years. THELEP later evaluated the results of implementation of standard MDT in control programmes, and confirmed its value in successfully dealing with the problem of drug-resistance in addition to ensuring effective cure.

Whereas the immediate problems of preventing drug resistance and improving effectiveness of treatment could be solved and sustained by means of research inputs from THELEP, progress towards primary prevention of leprosy by means of an anti-leprosy vaccine was relatively slow. Development of the first-generation vaccine, which was based on killed $M$. leprae, depended upon progress at several levels. First, sufficient quantities of M. leprae had to be collected from armadillos. Next, satisfactory purification procedures had to be established, tissue-free $M$. leprae obtained from armadillo tissues and the immunogenicity of the product in animals and humans had to be demonstrated. It then had to be shown that the vaccine provided protected in animal models, and lastly, field studies had to be carried out to demonstrate protection in human populations. IMMLEP went through all of these stages over a period of nearly 15 years, and ultimately demonstrated that armadillo-derived, killed $M$. leprae, in combination with BCG, was capable of protecting individuals to a high degree, as 
shown by the results of a field trial in India, although this could not be confirmed in two other trials, in Venezuela and Malawi.

At the same time, the 25 years since the establishment of IMMLEP have witnessed tremendous change, in terms both of scientific developments and of the global leprosy scene. First, with current trends in regulatory mechanisms, the possibility of licensing of an armadillo-derived product for routine use in humans appears rather limited. Moreover, developments in molecular biology, including sequencing the genome of M. leprae, have rendered the armadillo-derived product obsolescent. Further, there are serious doubts about the practicability of producing sufficient quantities of $M$. leprae from the armadillo.

However, the most important reason for the diminished interest in an anti-leprosy vaccine in recent years is the progress made toward eliminating leprosy by the widespread implementation of MDT. The number of registered cases in the world has decreased from over 5.4 million in 1985 to fewer than 700,000 recently. There has been an even more profound reduction of the number of smear-positive cases. These developments have raised the question of the cost-effectiveness of a vaccine, even assuming a high protective effect and good acceptability to the population. TDR itself, which originally strongly promoted development of a vaccine, appears to have reduced its priority for a leprosy vaccine. Last, a vaccine that can be used routinely in the field requires validation on a large scale in more than one situation. Whereas it was once possible to carry out trials of a killed M. leprae vaccine, it will be extremely difficult to carry out a trial of a new sub-unit vaccine, mainly because of the extremely limited number of suitable field areas and competent epidemiologists available to undertake trials lasting up to 10 years. Thus, as far as leprosy elimination is concerned, there appears to be no alternative to MDT, even if it has been slow to break the chain of transmission of $M$. leprae in the community.

Even recognizing the role of leprosy research so far in leprosy elimination, there is clearly a need for further research toward solving the remaining problems in leprosy. These include a better understanding of subclinical infection, particularly in relation to persistence of the disease in certain situations, and exploring possible extra-human reservoirs in nature. Apart from these, research efforts are needed in areas related to better quality of treatment for the patients, including prevention and management of nerve damage and reactions. In addition, it should be possible further to improve the MDT regimens. A last area for research is that of applied research dealing with more efficient application of the available tools for diagnosis, treatment and rehabilitation.

In conclusion, it is clear that leprosy research and leprosy elimination have complemented each other extremely well. Leprosy has also been an excellent model for the transfer of technology to the field, and simplification of technology for the benefit of patients and disease-control. I expect that, irrespective of the progress made toward leprosy elimination to date, leprosy research will continue to receive reasonable attention toward solving the remaining problems. This is not the time to reduce the emphasis on leprosy research. 